\title{
Modelling of Critical Current Density of Sintered Ag Added Bulk $\mathrm{MgB}_{2}$
}

\author{
M. SANTOSH* \\ E300 Huron Str., M5S 3J6, Faculty of Arts and Science, University of Toronto, Canada
}

(Received January 16, 2016; in final form May 25, 2016)

\begin{abstract}
Magnetic field dependence of critical current density $\left(J_{\mathrm{c}}\right)$ of bulk $\mathrm{MgB}_{2}$ material at $20 \mathrm{~K}$ is reported. The selected sintered $\mathrm{MgB}_{2}$ materials contained various amounts of silver. The silver added samples possess always a higher critical current density than the silver free ones. The critical current density increased with silver content and continuously decreased with increase of magnetic field. Further, the normalized volume pinning force density, $f_{\mathrm{p}}=F_{\mathrm{p}} / F_{\mathrm{p}, \max }$, versus reduced field $h=H_{\mathrm{a}} / H_{\text {irr }}$ for silver free and silver added samples suggested that grain boundary pinning is dominant. In essence, the model of thermal activated flux motion was successfully applied to fit the critical current density of the $\mathrm{MgB}_{2}$ materials.
\end{abstract}

DOI: 10.12693/APhysPolA.129.1197

PACS/topics: 74.72.-h, 74.25.Sv, 74.20.-z

\section{Introduction}

$\mathrm{MgB}_{2}$ is a simple ionic binary compound with hexagonal structure, superconducting up to $39 \mathrm{~K}[1]$. $\mathrm{MgB}_{2}$ was first synthesized in 1953 and Nagamatsu et al. discovered its superconducting properties in 2001 [2]. The family of the related compounds is characterized by an intermediate but still quite a high $T_{\mathrm{c}}$ and does not obtain intrinsic obstacles for current flow between grains [3, 4].

Development of $\mathrm{MgB}_{2}$ had strongly progressed due to advances in processing, characterization, and applications [5-8]. Two important parameters, namely upper critical magnetic field, $B_{\mathrm{c} 2}$, and critical current density, attain high values compared to conventional superconductors $[9,10]$. As a result, $\mathrm{MgB}_{2}$ receives a significant space for its applications in various technical and industrial areas. The examples are bulk magnets for magnetic resonance imaging (MRI), nuclear magnetic resonance (NMR), motors, energy storage systems, and non-contact bearings for liquid pumping purposes $[6,7,11-13]$. These applications are possible even in a polycrystalline state, which significantly simplifies the preparation.

On the occasion of taking a part in Shibaura Institute of Technology Internship Program and in Science Fair Project, I could study properties of $\mathrm{YBa}_{2} \mathrm{Cu}_{3} \mathrm{O}_{y}$ and $\mathrm{GdBa}_{2} \mathrm{Cu}_{3} \mathrm{O}_{y}$ superconductors in relation to guidance forces, levitation, modeling of critical current density etc. [14-16]. Also, I prepared $\mathrm{MgB}_{2}$ material and characterized it for use as a permanent magnet. In the technological and characterization process, the differential thermal analysis (DTA), X-ray difractometry (XRD), optical microscopy, and levitation force measurement techniques were utilized. Moreover, I had prepared silver free and silver added samples to enhance the performance

*e-mail: santoshjuly@msn.com of bulk material in which will be essential for industrial applications.

In this paper, we present results of magnetization measurements of selected silver free and silver added sintered bulk $\mathrm{MgB}_{2}$ samples performed at $20 \mathrm{~K}$. From the magnetization data, the critical current density and flux pinning performance were estimated. Further, the curves of critical current density as a function of magnetic field were interpreted in terms of thermally activated flux creep model.

\section{Experimental}

The $\mathrm{MgB}_{2}$ samples were fabricated using the in situ solid state reaction. Furuuchi Chemical Corporation had provided purified powders of amorphous B with 99\% purity, 300 meshes, and $\mathrm{Mg}$ metallic powder with 99\% purity, 200 meshes. These high-purified powders were mixed in the stoichiometric ratio of $1: 2(\mathrm{Mg}: \mathrm{B})$. A sterilized metallic $\mathrm{Ag}$ was added to the $\mathrm{MgB}_{2}$ powder mixtures in amounts of 0,4 , and $10 \mathrm{wt} \%$. Each resultant powder mixture was thoroughly grinded in glove box in Ar atmosphere (see Fig. 1, left). Uniaxial pressing machine was utilized to compress powder mixture into pellets with dimensions of $7 \mathrm{~mm}$ width and $20 \mathrm{~mm}$ diameter (see Fig. 1, middle). After all, the pellets were wrapped into tantalum foils and heated under $\mathrm{Ar}$ atmosphere in the tube up to the sintering temperature of $775^{\circ} \mathrm{C}$ (see Fig. 1, right). Finally, temperature was reduced to room temperature with the cooling rate of $100^{\circ} \mathrm{C} / \mathrm{h}$, the more details for the processing of these materials are reported elsewhere [17]. The microstructure of these samples was studied with a scanning electron microscope (SEM) and chemical compositions were analyzed by energy dispersive X-ray spectroscopy (EDX). Small specimens with dimensions of $1.5 \times 1.5 \times 0.5 \mathrm{~mm}^{3}$ were cut from bulk $\mathrm{MgB}_{2}$ samples and subjected to the measurements of the critical temperature $\left(T_{\mathrm{c}}\right)$ and magnetization hysteresis loops $(M-H$ loops $)$ in applied magnetic fields from -1 to $+5 \mathrm{~T}$ at $77 \mathrm{~K}$ using a SQUID magnetometer 
(Quantum Design, model MPMS5). $J_{\mathrm{c}}$ values were estimated using the extended Bean critical state model [18], formula for a rectangular isotropic sample given by

$$
J_{\mathrm{c}}=20 \Delta M /\left[a^{2} d(b-a / 3)\right],
$$

where $a, b$ are cross-sectional dimensions, $b \geq a$, and $\Delta M$ is the difference of magnetization on the increasing and decreasing field branch of the $M-H$ loop. $M$ is the volume magnetization, $M=m / V$, where $m$ is the measured moment and $V=a b d$ is the sample volume, $d$ is the sample thickness.

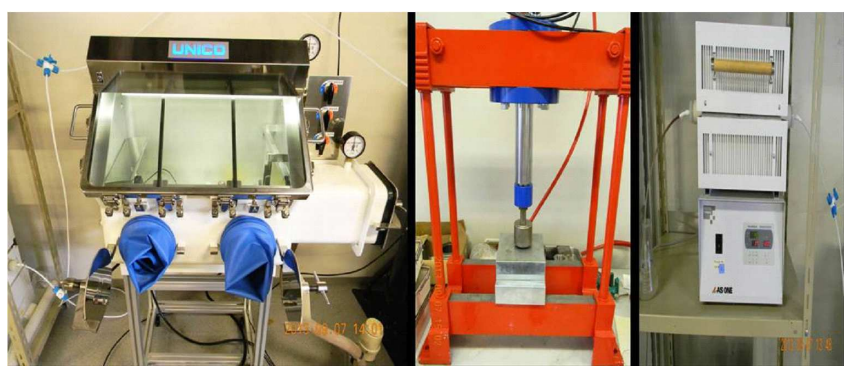

Fig. 1. Facilities used for the bulk $\mathrm{MgB}_{2}$ material preparation. Mixing the $\mathrm{MgB}_{2}$ powders was done in the glove box in Ar atmosphere (left); powder mixture was pressed into pellets by a uniaxial press (middle); heating of the bulk $\mathrm{MgB}_{2}$ material in $\mathrm{Ar}$ atmosphere was performed in the tube (right).

\section{Results and discussion \\ 3.1. Magnetization experiments}

In order to estimate the superconducting transition for the silver free and silver added $\mathrm{MgB}_{2}$ materials, $M-T$ data were collected between $15 \mathrm{~K}$ and $50 \mathrm{~K}$ using a SQUID magnetometer (Quantum Design, model MPMS5). Figure 2 (left) presents the temperature dependence of the dc susceptibility in the zero field cooled process in magnetic field of $1 \mathrm{mT}$. Both silver free and silver added $\mathrm{MgB}_{2}$ samples were sintered at $775^{\circ} \mathrm{C}$ in $\mathrm{Ar}$ atmosphere. All samples exhibited a sharp superconducting transition around 38.5-38.7 K (see Fig. 2, left). The superconducting transition was only $0.7 \mathrm{~K}$ wide, which indicates good quality of the samples. The reported superconducting transitions are similar or little bit higher than those of the recently reported bulk $\mathrm{MgB}_{2}$ materials produced by the same, sintering technique $[19,20]$. The silver addition did not deteriorate the superconducting properties of the $\mathrm{MgB}_{2}$ bulks, similar to the bulk melt-processed $\mathrm{REBa}_{2} \mathrm{Cu}_{3} \mathrm{O}_{y}$ materials.

The $M-H$ data of the silver free and silver added bulk $\mathrm{MgB}_{2}$ materials were collected at $20 \mathrm{~K}, H \| c$-axis using a SQUID magnetometer (see inset in Fig. 2, right). It is clear that emu was improved for silver added samples as compared to silver free sample. Moreover, one can note that magnetization decreased with increase of the magnetic field. To understand further on bulk $\mathrm{MgB}_{2}$ performance, especially the silver addition, the critical current density $\left(J_{\mathrm{c}}\right)$ was estimated using the extended Bean critical state model as shown in Fig. 2 (right). The critical current density decayed with increasing magnetic


Fig. 2. The superconducting transition in terms of the temperature dependence of the DC magnetic susceptibility left) and the $J_{\mathrm{c}}\left(H_{\mathrm{a}}\right)$ dependence in the semilogarithmic scale (right). Insert of right figure represents the $\mathrm{MH}$ loops at $20 \mathrm{~K}$ ( $H \| c$-axis) for all three $\mathrm{MgB}_{2}$ samples investigated.

field, the decay rate depending on the silver addition (see in Fig. 2, right). In the sample with $4 \mathrm{wt} \% \mathrm{Ag}$, the remnant $J_{\mathrm{c}}$ value was the highest, around $292 \mathrm{kA} / \mathrm{cm}^{2}$ $(H \| c$-axis, $20 \mathrm{~K})$. The $J_{\mathrm{c}}$ values in applied magnetic fields were $210 \mathrm{kA} / \mathrm{cm}^{2}$ at $0.5 \mathrm{~T}$ and $115 \mathrm{kA} / \mathrm{cm}^{2}$ at $1 \mathrm{~T}$. The critical current densities determined for the silver added sample are quite high as compared to the silver free sample and also somewhat higher than the values for $\mathrm{Ag}$-added bulk $\mathrm{MgB}_{2}$ materials. Kumar et al. prepared Ag-doped $\mathrm{MgB}_{2}$ bulk superconductors by solid state sintering process i.e. $6 \mathrm{~h}$ at $850^{\circ} \mathrm{C}$ and noticed an important advantage which is the prevention/reduction of loss of $\mathrm{Mg}$ and this is the main problem which is commonly observed in sintering of $\mathrm{MgB}_{2}$ bulk samples [21]. In addition, the critical current density for the silver added samples are improved as compared to the silver free sample, where the sample added $5 \mathrm{wt} \%$ of silver showed highest critical current density $40 \mathrm{kA} / \mathrm{cm}^{2}$ at $20 \mathrm{~K}$. These values are very low as compared to the present results which clearly indicates that both optimum silver content and optimum sintering temperature are crucial to get high performance bulk $\mathrm{MgB}_{2}$ material. Similarly, improvements had reported that $\mathrm{Ag}$ doping significantly enhances $\mathrm{MgB}_{2}$ formation rate in $\mathrm{MgB}_{2}$ wires with enhanced properties [22]. On the other hand, these results indicate that $\mathrm{Ag}$ addition to $\mathrm{MgB}_{2}$ can be one of the ways to improve not only mechanical properties [17, 21-24] but also flux pinning; the critical temperature remains almost unchanged. It is also positive that $\mathrm{Ag}$ does not react with $\mathrm{MgB}_{2}$ and can help to reduce cracks density like in cuprates [25]. More recently, EDX analysis by TEM had lucidly confirmed that $\mathrm{Ag}$ enriched phase distributed in $\mathrm{MgB}_{2}$ matrix obtains a composition in close to $\mathrm{AgMg}_{3}[21]$ and its size and dispersion in $\mathrm{MgB}_{2}$ matrix is somewhat dependent on initially added silver content. Further, it was also confirmed by AFM observations that nanometer sized $\mathrm{AgMg}_{3}$ particles were dispersed in $\mathrm{MgB}_{2}$ matrix [17]. Therefore, it is essential to estimate the flux pinning behavior of bulk $\mathrm{MgB}_{2}$ material with $\mathrm{Ag}$ addition and more details are presented below. 


\subsection{Flux pinning analysis of $\mathrm{Ag}$ added $M g B_{2}$}

In order to estimate and comprehend the flux pinning behavior of bulk $\mathrm{MgB}_{2}$ materials with $\mathrm{Ag}$ addition, sintered for $3 \mathrm{~h}$ at $775^{\circ} \mathrm{C}$, the normalized volume pinning force density, $f_{\mathrm{p}}=F_{\mathrm{p}} / F_{\mathrm{p} \text {,max }}$, was plotted versus the reduced field, $h=H_{\mathrm{a}} / H_{\text {irr }}$ at $20 \mathrm{~K}$. The irreversibility field, $H_{\text {irr }}$, was determined from magnetization loops. The resulting $f_{\mathrm{p}}(h)$ curves for the $\mathrm{MgB}_{2}$ samples with $\mathrm{Ag}$ free, $4 \mathrm{wt} \%$ and $10 \mathrm{wt} \%$ of $\mathrm{Ag}$ added samples are shown in Fig. 3. The scaling works efficiently for all samples irrespective of $\mathrm{Ag}$ with a peak located at $h_{0} \approx 0.21$ which suggests that dominant pinning centers were extended with normal-conducting defects. Similar values were obtained in earlier reports presented in the literature for small $\mathrm{MgB}_{2}$ samples [20, 26, 27]. For instance, the peak position around $h_{\max }=0.2$ suggests that grain-boundary pinning were active for both set of samples. Consequently, these results clearly indicate that silver doping can enhance the critical current density at lower magnetic field without changing the flux pinning mechanisms.

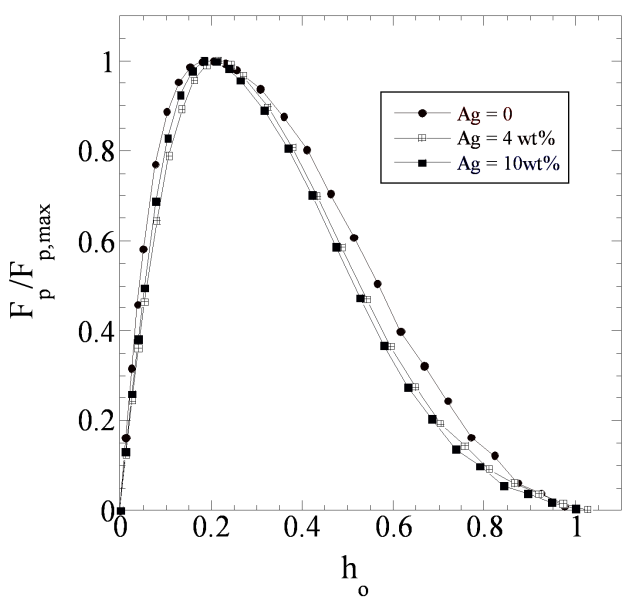

Fig. 3. The normalized volume pinning force density, $f_{\mathrm{p}}=F_{\mathrm{p}} / F_{\mathrm{p}, \max }$, was plotted against the reduced field, $h=H_{\mathrm{a}} / H_{\text {irr }}$ at $20 \mathrm{~K}$ for $\mathrm{MgB}_{2}$ superconductor with varying contents of silver. All samples were produced by sintering process at $775^{\circ} \mathrm{C}$ in argon atmosphere.

\subsection{Modeling the $J_{\mathrm{c}}-B$ curves}

Modeling the critical current density $\left(J_{\mathrm{c}}-H\right)$ curves of the bulk superconducting materials is a way to better understanding of the flux pinning mechanism(s) and improving the materials' performance. Up to now, several models have been proposed based on thermodynamic and some phenomenological considerations. For bulk high $T_{\mathrm{c}}$ superconductors with an intensive thermally activated flux motion Perkins et al. [28, 29] and Jirsa et al. proposed a model enabling to fit the secondary peak on the critical current density as a function of magnetic field [30]. It worked well for NEG-123 and Gd-123 bulks and for Eu-123 thick films [14]. Up to date, there is no data regarding modeling of critical current density of bulk $\mathrm{MgB}_{2}$ material and no secondary peak was observed in $\mathrm{MgB}_{2}$ due to a continuously decreasing dependence. Therefore, we attempted to apply for sintered bulk $\mathrm{MgB}_{2}$ materials using exponentially decaying function with the first term in the above approach and describing its central peak

$$
J_{\mathrm{c}}(B)=J_{\mathrm{c} 1} \exp \left(-B / B_{0}\right),
$$

where $J_{\mathrm{c} 1}$ is the remnant critical current density (at the self-field), $B$ stands for the applied magnetic field value, and $B_{0}$ is a fitting constant indicating how fast the peak decays with increasing magnetic field. We note that an exponentially decaying $J_{\mathrm{c}}(B)$ dependence was first observed in thin cuprate films by Zeldov and in other types of cuprate samples by other notable researchers.

\subsection{Fitting the critical current density of bulk $\mathrm{MgB}_{2}$ material}

For fitting the experimental curve of $\mathrm{MgB}_{2}$, we need to take into account of coherence length and thus also vortex core size are in $\mathrm{MgB}_{2}$ about 20 times larger than in cuprates. It means that also the relevant defects have to be much larger and can be hardly regarded as pointlike defects that are responsible for secondary peak in cuprates. In $\mathrm{MgB}_{2}$, only large defects are effective and these are known to contribute to the central maximum. Thus, only the first term of expression used for RE-123 materials [14] is effective for $\mathrm{MgB}_{2}$ as in Eq. (2). When fitting the experimental curve, we would have to consider the rather broad peak at zero magnetic field. Therefore, it is impossible to utilize pure exponential decay with an exact experimental maximum value for $J_{\mathrm{c} 1}$. Instead, a higher $J_{\mathrm{c}}$ is needed. On the other hand, in order to fit by pure exponential decay, we will need to skip the first 6-8 experimental points. For the silver free and $4 \mathrm{wt} \%$ silver added sintered bulk $\mathrm{MgB}_{2}$ material the $J_{\mathrm{c}}(B)$ fit by Eq. (2) is presented in Fig. 4. The fittings for both $\mathrm{MgB}_{2}$ materials were quite successful. The best fitting for silver free $\mathrm{MgB}_{2}$ material was

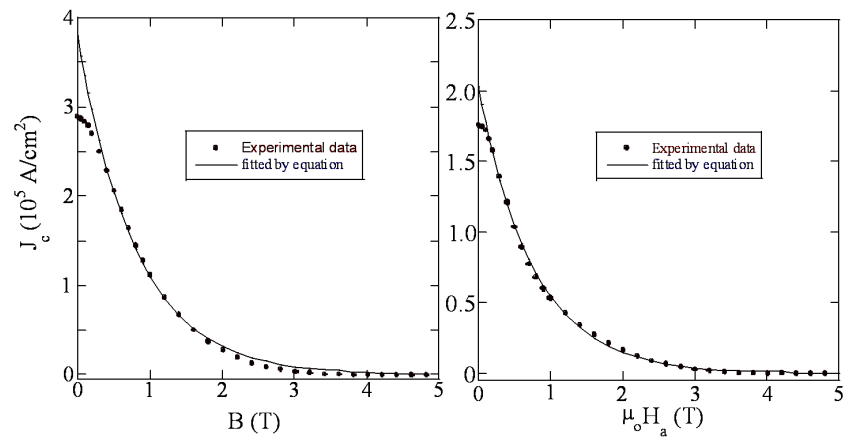

Fig. 4. The experimental data (symbols) were fitted by Eq. (2) (the full lines) for silver free $\mathrm{MgB}_{2}$ data (left) and the $4 \mathrm{wt} \%$ of silver added $\mathrm{MgB}_{2}$ material (right).

obtained with $J_{\mathrm{c} 1}=203 \mathrm{kA} / \mathrm{cm}^{2}$ and $B_{0}=0.81 \mathrm{~T}$. For the $\mathrm{MgB}_{2}$ material with $4 \mathrm{wt} \% \mathrm{Ag}$, the fitting parameters were $J_{\mathrm{c} 1}=380 \mathrm{kA} / \mathrm{cm}^{2}$ and $B_{0}=0.76 \mathrm{~T}$. 


\section{Summary}

In conclusion, the critical current density of sintered bulk $\mathrm{MgB}_{2}$ material was estimated at $20 \mathrm{~K}$ by utilizing magnetization experiments on SQUID magnetometer. Normalized volume pinning force density, $f_{\mathrm{p}}=$ $F_{\mathrm{p}} / F_{\mathrm{p} \text {,max }}$, versus reduced field $h=H_{\mathrm{a}} / H_{\text {irr }}$ for both silver free and silver added samples clearly indicated that grain boundary pinning is dominant. Further, the results were modeled using the exponentially decaying function of magnetic field, Eq. (2). This function fitted well for the experiment data in both types of material: with and without silver addition. The best fit for silver free $\mathrm{MgB}_{2}$ was with $J_{\mathrm{c} 1}=203 \mathrm{kA} / \mathrm{cm}^{2}$ and $B_{0}=0.81 \mathrm{~T}$, whereas for $\mathrm{MgB}_{2}$ with $4 \mathrm{wt} \% \mathrm{Ag}$ the best fit was obtained with $J_{\mathrm{c} 1}=380 \mathrm{kA} / \mathrm{cm}^{2}$ and $B_{0}=0.76 \mathrm{~T}$. With further increase of silver content $J_{\mathrm{c}}$ started to decrease. Therefore, according to the present study the optimum content of silver is around $4 \mathrm{wt} \%$. The experimental temperature was chosen as a potential compromised operation temperature for applications. Our results show that this material has a great potential for use in practice.

\section{Acknowledgments}

I would like to thank Professor M. Murakami, President of Shibaura Institute of Technology (SIT), for giving me an opportunity to work at Superconductivity Research Laboratory, SIT. Special thanks to Dr. Milos Jirsa (Institute of Physics, ASCR) for his valuable suggestions and advising this project. Lastly, I would like to thank my loving family members for their endless encouragement and love.

\section{References}

[1] J. Kortus, I.I. Mazin, K.D. Belashchenko, V.P. Antropov, L.L. Boyer, Phys. Rev. Lett. 86, 4656 (2001).

[2] R.J. Nagamatsu, N. Nakagawa, T. Muranaka, Y. Zenitani, J. Akimitsu, Nature 410, 63 (2001).

[3] B.A. Glowacki, N. Majoros, M. Vickers, J.E. Evetts, Y. Shi, I. McDougall, Supercond. Sci. Technol. 14, R115 (2001).

[4] G. Giunchi, G. Ripamonti, T. Cavallin, E. Bassani, Cryogenics 46, 237 (2006).

[5] K. Kajikawa, T. Nakamura, IEEE Trans. Appl. Supercond. 19, 1669 (2009).

[6] K. Vinod, R.G. Abhilash Kumar, U. Syamaprasad, Supercond. Sci. Technol. 20, R1 (2007).

[7] E. Perini, G. Ginuchi, Supercond. Sci. Technol. 22, 045021 (2009).

[8] M. Muralidhar, A. Ishihara, K. Suzuki, Y. Fukumoto, Y. Yamamoto, M. Tomita, Physica C 494, 85 (2013).
[9] S.X. Dou, S. Soltanian, J. Horvat, X.L. Wang, S.X. Zhou, M. Ionescu, H.K. Liu, P. Munroe, M. Tomsic, Appl. Phys. Lett. 81, 3419 (2002).

[10] J.H. Kim, S. Zhou, M.S.A. Hossain, A.V. Pan, S.X. Dou, Appl. Phys. Lett. 89, 142505 (2006)

[11] J.H. Durrell, C.E.J. Dancer, A. Dennis, Y. Shi, Z. Xu, A.M. Campbell, N. Hari Bau, R.I. Todd, C.R.M. Grovenor, D.A. Cardwell, Supercond. Sci. Technol. 25, 112002 (2012).

[12] M. Muralidhar, K. Inoue, M.R. Koblischka, M. Tomita, M. Murakami, J. Alloys Comp. 608, 102 (2014).

[13] A. Yamamoto, A. Ishihara, M. Tomita, K. Kishio, Appl. Phys. Lett. 105, 032601 (2014).

[14] M. Santosh, Acta Phys. Pol. A 126, 808 (2014).

[15] M. Santosh, M.R. Koblischka, Europ. J. Phys. Educ. 5, 1 (2014).

[16] M. Santosh, in: Oxide Thin Films, Multilayers, and Nanocomposites, Eds. P. Mele, T. Endo, S. Arisawa, C. Li, T. Tsuchiya, Springer, 2015, p. 97.

[17] M. Muralidhar, K. Inoue, M.R. Koblischka, M. Murakami, J. Adv. Eng. Mater. 17, 831 (2015).

[18] D. Chen, R.B. Goldfarb, J. Appl. Phys. 66, 2489 (1989).

[19] T. Naito, T. Sasaki, H. Fujishiro, Supercond. Sci. Technol. 25, 095012 (2012).

[20] M. Muralidhar, K. Nozaki, H. Kobayashi, X.L. Zeng, A. Koblischka Veneva, M.R. Koblischka, K. Inoue, M. Murakami, J. Alloys Comp. 649, 833 (2014).

[21] D. Kumar, S.J. Pennycook, J. Narayan, H. Wang, A. Tiwari, Supercond. Sci. Technol. 16, 455 (2003).

[22] K. Yamamoto, K. Osamura, S. Balamurugan, T. Nakamura, T. Hoshino, I. Muta, Supercond. Sci. Technol. 16, 1052 (2003).

[23] C.E. Cunningham, C. Petrovic, G. Lapertot, S.L. Bud'ko, F. Laabs, W. Straszheim, D.K. Finnemore, P.G. Canfield, Physica $C$ 353, 5 (2001).

[24] J. Shimoyama, K. Hanafusa, A. Yamamoto, Y. Katsura, S. Horii, K. Kisho, H. Kumakura, J. Phys. Conf. Ser. 97, 012255 (2008).

[25] J.-C. Grivel, A. Abrahamsen, J. Bednarcik, Supercond. Sci. Technol. 21, 035006 (2008).

[26] V. Sandu, Mod. Phys. Lett. 26, 1230007 (2012).

[27] M.R. Koblischka, A. Wiederhold, M. Muralidhar, K. Inoue, T. Hauet, B. Douine, K. Berger, M. Murakami, U. Hartmann, IEEE Trans. Magn. $\mathbf{5 0}$ 9000504 (2014)

[28] G.K. Perkins, A.D. Caplin, Phys. Rev. B 51, 8513 (1995).

[29] G.K. Perkins, A.D. Caplin, Phys. Rev. B 54, 12551 (1996).

[30] M. Jirsa, L. Pust, D. Dlouhy, M.R. Koblishchka, Phys. Rev. B 55, 3276 (1997). 